\title{
Analyzing 60 GHz Radio Links for Indoor Communications
}

\author{
Jing Wang, Student Member, IEEE, R Venkatesha Prasad, Member, IEEE, and Ignas Niemegeers
}

\begin{abstract}
With the increase in the capacity of mobile communication devices, it is but natural to expect that these devices would work ubiquitously anywhere anytime to offer high data rate support. Recently $60 \mathrm{GHz}$ frequency band has been identified as an obvious choice for the high data rate indoor communications. However, as the $60 \mathrm{GHz}$ radio system relies on line-of-sight (LOS) transmission for achieving Gbps data rate, the communication can be easily interrupted by obstructions breaking the LOS link, which happens often due to the movement of people in a typical indoor environment. In this paper, we define and present an analytical model for assessing link stability of $60 \mathrm{GHz}$ radio for indoor wireless networks. We have developed a raybased model to calculate the shadowing loss caused by the presence of people around the communication link while taking into account the indoor channel characteristics of 60 $\mathrm{GHz}$ radio and the antenna configuration. We have further considered different types of mobility of people with the raybased model of $60 \mathrm{GHz}$ link to obtain a holistic link stability model in realistic scenarios. We have given examples to show the relevance of our model and its applicability using both simulation and numerical evaluations ${ }^{l}$.
\end{abstract}

Index Terms - $60 \mathrm{GHz}$ radio, link stability, line-of-sight, human body shadowing

\section{INTRODUCTION}

The evolution of multimedia services coupled with the increase in capability of communication devices evokes a proliferation of high data rate indoor multimedia applications and services, such as HDTV, 3D-Games, virtual conferencing, etc. These applications require high bandwidth, for example, HDTV requires up to $3 \mathrm{Gbps}$ for the raw signal and $80 \mathrm{Mbps}$ for MPEG-4 compressed signal [1] [2]. This is far beyond the capability of the current offthe-shelf WLAN/WPAN systems. Research and studies on $60 \mathrm{GHz}$ radio system [3] [4] [5] have created an opportunity to support gigabit speed on wireless communication links within a short distance, less than $10 \mathrm{~m}$ in general. Therefore, a $60 \mathrm{GHz}$ radio network is a promising candidate to enable these demanding indoor multimedia applications to go onthe-air in the very near future.

By nature the $60 \mathrm{GHz}$ radio wave experiences severe oxygen absorption; therefore directional antenna has been

\footnotetext{
${ }^{1}$ This work is supported in by Dutch IOP GemCom project "Future Home networks".

Authors are with the Faculty of Electrical Engineering, Mathematics and Computer Science (EEMCS), Delft University of Technology, 2628 CD Delft, The Netherlands, e-mail: \{Jing.Wang, R.R.VenkateshaPrasad, I.G.M.M.Niemegeersg\}@tudelft.nl.
}

Contributed Paper

Manuscript received October 12, 2009 recommended to provide line-of-sight (LOS) links to increase energy efficiency. However, a large amount of energy is lost when $60 \mathrm{GHz}$ radio wave is obstructed. Thus LOS is very sensitive to the environment, especially, when people are moving around. More than $20 \mathrm{~dB}$ attenuation has been observed during the field measurements [6] when a person moves across LOS links. Commonly in WLAN/WPAN scenarios, the movement of people thereby obstructing the communication links is unavoidable. In many cases, people are the important elements of the network as they use and carry the network devices. This is a very important aspect to be considered while designing high speed $60 \mathrm{GHz}$ wireless networks supporting stringent QoS guarantees required for these multimedia applications.

In this paper, we introduce a link stability model - for a given link quality requirement, e.g., the packet delivery ratio, to reflect on the capability of the link to continuously sustain the requirement for a certain duration. This model has been specifically built by taking into account the indoor channel characteristics of $60 \mathrm{GHz}$ radio, the antenna configuration and the mobility pattern of people. A ray-based model has been developed to characterize the $60 \mathrm{GHz}$ radio propagation from the transmitter antenna to the receiver antenna. It enables the analysis of instantaneous shadowing due to the presence of people around the transceiver pair, and further to calculate the power loss due to shadowing. The indoor mobility of people has been investigated for understanding the frequency with which people walk into the LOS link and consequently the duration of shadowing that leads to link failures. We employ both the simulation and analytical approaches. Simulation uses the random way point (RWP) mobility model and the analytical approach uses certain simplifications to derive the distribution of the duration of link failure. This study of the link stability is expected to help in designing and evaluating various protocols and algorithms; for example, it can be used as a parameter representing or quantifying the behavior of these LOS links to help vertical handover decision making, or it can be further formulated as the routing metric for path selection.

The rest of the paper is organized as follows. In Section II we define link stability with a brief overview of the related works. Section III introduces the ray-based model for 60 $\mathrm{GHz}$ link, which is able to take into account the shadowing loss due to people blocking $60 \mathrm{GHz}$ radio wave propagation paths. The calculation of shadowing loss because of people walking across is discussed in detail in Section IV. Section $\mathrm{V}$ presents the link stability analysis based on the specific indoor mobility models of people, and Section VI concludes this article. 


\section{EARLIER STUdies AND LinK STABILITY DEFINITION}

\section{A. Earlier Studies}

Early studies on the evaluation of link stability, in general, were mainly based on two types of information. Signal strength information as proposed in [7] is used for the signal stability based adaptive routing (SSA) where, if a node receives a strong signal from a neighbor then the link between them is considered to be stable, otherwise the link is considered as unstable. An enhanced version was proposed in [8] wherein, instead of directly looking into the received signal strength, the difference between the current and the previous signal strength measurements of the link is considered. If the difference is positive the link is considered as a stable link. The other approach is based on the number of consecutive pilot signals [9]. That is, letting all nodes send pilot signals periodically and if the number of consecutive pilot signals received from a neighboring node exceeds a certain threshold, then the link is considered as stable.

In the context of MANETs, the studies on link stability mostly focus on calculating the link survival time under network topology changes considering specific mobility models for networked devices. Authors in [10] [11] developed the metrics for characterizing link stability based on the analysis of durations of link uptime in several mobility scenarios. The link stability metrics are further adopted in routing algorithms as an important parameter while selecting the preferred routes [12] [13] [14]. However, as mentioned earlier, the networks using 60 $\mathrm{GHz}$ radio communication links may break even when devices are stationary but when there is an obstacle in the path of LOS links. The influence of the human activity on $60 \mathrm{GHz}$ indoor radio channel has been studied extensively based on the field measurements. In [6], it is found that when a person moves between the fixed $60 \mathrm{GHz}$ radio terminals, variations in the received signal envelope are caused. The rate of fluctuation increases significantly with mo-tion; and the fading statistics depends on the speed, the number of persons, the body size and the propagation environment. In [15], the measurement results confirm that human bodies are significant obstacles for $60 \mathrm{GHz}$ radio wave propagation. The duration of the consequent shadowing increases with the number of persons within the environment, but the amplitude of the shadowing only depends on the configuration of antennas. Mathematical models of the human body shadowing in the indoor radio systems have been investigated in [16] [17] [18], where the body is typically modeled as a cylinder with the average height of people and the diameter as the average shoulder width of people. We try to use some of the ideas from these earlier works to define and model the link stability of $60 \mathrm{GHz}$ links. This result will be helpful in characterizing these millimeter wave links to see whether they can support high data rate media. We first introduce the term link stability.

\section{B. Link Stability of the $60 \mathrm{GHz}$ Radio Communication Link}

All the above studies on the $60 \mathrm{GHz}$ radio links have focused on the received signal variation due to the movement of people across the communication link. However, from the point of view of the network, we are more interested in the statistical properties of the link. More specifically, the duration and interval of the shadowing events that cause the significant received signal power degradation so that the link is not able to sustain the communication with a certain required quality. Such link statistics are of great importance for estimating network performance. For example, estimation of packet error rate with the burst packet loss due to the absence of the LOS transmission. The burst loss ratio at any burst length can be obtained with the knowledge of the frequency of occurrence of significant shadowing which has the duration of the burst length considered. Therefore, we are concentrating on developing a link stability model, which statistically reflects whether the link is able to continuously sustain the link quality requirements, for example, throughput, delay bounds, packet delivery ratio etc., for certain period of time.

Here we define link stability as the probability of occurrence of a link failure in a given duration. Link failure, here, means that the link quality is below the predefined link quality threshold for guaranteeing an end-to-end QoS. Since in most scenarios using $60 \mathrm{GHz}$ radio communications we employ only stationary devices, here we consider link failure mainly as a result of the major radio propagation paths being blocked by people, which leads to a significant overall shadowing, and consequently the received signal power is not enough for correctly detecting the information. Based on this approach, we discern a link having better stability only if the link has a higher probability that the link failure duration is shorter than the time period for transmitting a packet of certain length.

\section{Ray-Based Model of 60 GHz Radio}

The link quality is fundamentally determined by the underlying radio system in use. Therefore, we first discuss a ray-based model for characterizing the $60 \mathrm{GHz}$ radio propagation towards the receiver. Comparatively, the generally used models such as lognormal path loss model, kray model [19] and impulse response model [20] focus on the path loss phenomena on the average, whereas our ray-based model is able to analyze the instantaneous shadowing effect that blocks certain propagation paths of the $60 \mathrm{GHz}$ radio wave due to the presence of people around the transceivers.

\section{A. Angular Power Density Model of $60 \mathrm{GHz}$ Channel}

For the $60 \mathrm{GHz}$ radio applications in indoor environments, it is highly likely that the transmitter and the receiver of a single hop link are located in the same room to avoid high penetration loss caused by construction materials. In this case, the received power consists of contribution from two parts the incoming radio wave from the LOS path and the multipath waves (except LOS directions) that are mainly composed of the waves scatted and reflected by walls, furniture, floor, ceiling, etc. The Rician K-factor is commonly used to characterize the influence of the multipath waves to the LOS 
wave. It is defined as the ratio of the power from the LOS wave to the power of all the multipath waves, i.e., $K=\left|\alpha_{0}\right|^{2} / \sum_{n=1}^{N}\left|\alpha_{n}\right|^{2}$, where $\alpha_{0}$ is the amplitude of the LOS wave and $\alpha_{n}$ is the amplitude of the $n$-th scattered wave.

Based on the previous studies on $60 \mathrm{GHz}$ indoor channels, the received signal power can further be modeled by two primary profiles: (1) the power decay profile (PDP), which characterizes power density of the incoming waves on a time scale, and (2) the angular spreading profile (ASP), which characterizes the angular distribution of the incoming waves at any time.

According to the measurement in [21], at $60 \mathrm{GHz}$ the PDP shape of a Rician channel can be mathematically expressed as follows,

$$
P_{P}(\tau)= \begin{cases}0, & \tau<0, \\ \left|\alpha_{0}\right|^{2} \delta(\tau), & \tau=0, \\ \Pi, & 0<\tau \leq \tau_{c}, \\ \Pi \cdot e^{-\gamma\left(\tau-\tau_{c}\right)}, & \tau>\tau_{c} .\end{cases}
$$

where $\alpha_{0}$ is the amplitude of the LOS wave, $\sqrt{\Pi}$ is the amplitude of a constant part with duration $\tau_{c}, \Pi=\left|\alpha_{0}\right|^{2} \gamma / K$ with $\gamma$ being the decay factor. This constant part is observed in some cases where the directional antennas of the transmitter and the receiver are marginally misaligned. When $\tau_{c}=0$, $P_{P}(\tau)$ becomes the commonly applied exponentially decaying channel model.

The ASP of the incoming wave at any time $\tau$ can be expressed in several ways. A simplified model has been discussed in [22], where the incoming scattered waves were considered to be uniformly distributed in the surrounding space (a sphere surface) of the receiving antenna during the receiving period, which is denoted by $\tau_{\max }-$ the maximal delay of the multipath waves. For waves coming after $\tau_{\max }$, the power of those multipath waves is too weak to be detected from the noisy background due to the energy loss during reflection and scattering on the path. However, as argued in [23] it is more sensible to consider the angular spread varying with time, more specifically, the angular spreading increases with delay. We assume that at any time $\tau \leq \tau_{\max }$ the angles of the incoming waves are uniformly distributed in the incident area of $\Omega=\left\{\phi \in\left[\phi_{\tau}^{\min }, \phi_{\tau}^{\max }\right], \theta \in\left[\theta_{\tau}^{\min }, \theta_{\tau}^{\max }\right]\right\}$, which is a sphere cap surface centered at the receiver site, as illustrated in Fig. 1. This surface area increases proportionally with delay till $\tau_{\max }$ and reaches the maximal spreading area of size $S_{\max }$. As discussed in [23], the root mean square (RMS) angular spreading for $802.15 .3 \mathrm{a}$ UWB channel is $32^{\circ}$ in both elevation and azimuth planes. Using the spherical coordinate system in Fig. 1, where the LOS direction is along x-axis, the ASP is expressed as,

$$
P_{A}(\phi, \theta, \tau)= \begin{cases}\delta(\tau), & \tau=0, \phi=0, \theta=\pi / 2, \\ \frac{\tau_{\max }}{\tau S_{\max }}, & 0<\tau \leq \tau_{\max }, \phi \neq 0, \theta \neq \pi / 2 .\end{cases}
$$

At any point $P:(\phi, \theta)$ on the incident area (see Fig. 1), the time of the first arrival of scattered waves is, $\mathrm{T}=\tau_{\max } S(\phi, \theta) / S_{\max }$, where $S(\phi, \theta)$ is the size of the sphere cap surface with the point $P:(\phi, \theta)$ on the edge, and it can be calculated as $S(\phi, \theta)=2 \pi(1-\sin (\theta) \cos (\phi))$.

We define angular power density (APD) as the spatial distribution of the power of the incoming radio waves at the receiver during $\tau_{\max }$. It is calculated as,

$$
\begin{aligned}
P_{D}(\phi, \theta) & =\int_{0}^{\tau_{\max }} P_{A}(\phi, \theta, \tau) P_{P}(\tau) d \tau \\
& = \begin{cases}\left|\alpha_{0}\right|^{2}, & \phi=0, \theta=\pi / 2, \\
P_{D}^{\text {Scat }}(\phi, \theta), & \text { otherwise. }\end{cases}
\end{aligned}
$$

$P_{D}^{\text {Scat }}(\phi, \theta)$ is the APD for the scattered waves incident on the receiving antenna, Consider the transmitter and the receiver antennas are perfectly aligned, it is given by,

$$
\begin{aligned}
& P_{D}^{\text {Scat }}(\phi, \theta)=\int_{\mathrm{T}}^{\tau_{\max }} \frac{\tau_{\max }}{S_{\max } \tau} \frac{\left|\alpha_{0}\right|^{2}}{K} \gamma e^{-\gamma \tau} d \tau \\
& =\frac{\left|\alpha_{0}\right|^{2} \gamma \tau_{\max }}{S_{\max } K}\left(\ln \left|-\gamma \tau_{\max }\right|+\sum_{n=1}^{\infty} \frac{\left(-\gamma \tau_{\max }\right)^{n}}{n n !}-\ln |-\gamma \mathrm{T}|-\sum_{n=1}^{\infty} \frac{(-\gamma \mathrm{T})^{n}}{n n !}\right) .
\end{aligned}
$$

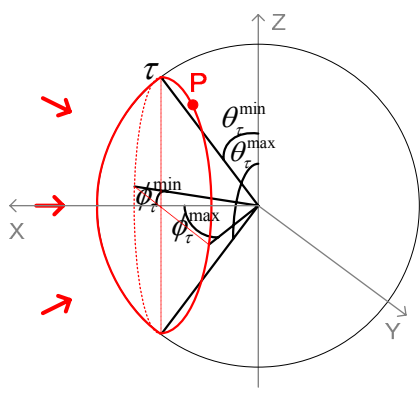

Fig. 1. Multipath waves angular spreading in $60 \mathrm{GHz}$ radio channel in the spherical coordinate system originated from the receiver site.

\section{B. Impact of Directional Antennas}

Antenna direction can be expressed in the form of antenna power pattern $A_{\Psi}(\Omega)$ which describes the shape of the lobe(s) in the spherical coordinate system having the position of the receiving antenna as the origin. The subscript $\Psi=(\psi, v)$ represents the direction of the antenna main lobe, and $\Omega=(\phi, \theta)$ represents the direction of the incoming waves in the spherical coordinate system. $\Omega_{0}=\left(\phi_{0}, \theta_{0}\right)$ denotes the direction of the incoming LOS wave. In this paper, we consider a single antenna with cosine-shaped power pattern, which is a good approximation of the power pattern for many types of elementary 
antennas, such as horn, patch and dipole antennas [22]. When the antenna main lobe direction aligns with the LOS direction, $\mathrm{x}$-axis in Fig. 1, i.e., $\Psi=(0,(\pi / 2))$, the 3 -dimensional power pattern is expressed in the spherical coordinate system as,

$$
A_{\Psi}(\Omega)=A(\phi, \theta)=2(2 q+1)(\sin (\theta) \cos (\phi))^{2 q},
$$

with ranges $\phi \in[-\pi / 2, \pi / 2]$ and $\theta \in[0, \pi]$. The parameter $q \geq 0$ is used to adjust the power pattern shape and is related to the half-power beam width (HPBW), which on the principal azimuth and elevation planes are the same in this pattern and expressed as $\sigma_{A}=2 \arccos \left(2^{-1 /(2 q)}\right)$. For example, when we take $q=2$, the cosine-shaped power pattern has HPBW of $65^{\circ}$.

Antenna directivity affects both radiated energy at the transmitter and the received power at the receiver. From the transmitter perspective, the antenna directionality is the consequence of scaling an isotropic energy source at each direction according to the antenna power pattern. The antenna gain is essentially determined by its power pattern with the relation $G=\varepsilon D$. Here $\varepsilon$ is the antenna efficiency and $D=4 \pi A_{\max } / P_{\text {rad }}$ is the antenna directivity. With our defined antenna power pattern, for a lossless antenna, i.e., $\varepsilon=1$, we have the antenna gain as,

$$
G=4 \pi \frac{A\left(0, \frac{\pi}{2}\right)}{\iint_{(\phi, \theta) \in \Omega} A(\phi, \theta) \sin (\theta) d \theta d \phi}=2(2 q+1) .
$$

Further, at the receiver due to antenna directivity the energy of the incoming waves is scaled differently according to the direction. In the spherical coordinate system with receiver position as the origin we update APD with (3) and (5) as,

$$
\begin{aligned}
& P_{D}^{\prime}(\phi, \theta)=P_{D}(\phi, \theta) A(\phi, \theta) \\
& =2(2 q+1) \times \begin{cases}\left|\alpha_{0}\right|^{2}, & \phi=0, \theta=\frac{\pi}{2}, \\
P_{D}^{S c a t}(\phi, \theta)(\sin (\theta) \cos (\phi))^{2 q}, & \text { otherwise. }\end{cases}
\end{aligned}
$$

Accordingly, received signal power can be obtained by integrating power density on the spherical area centered at the receiver as,

$$
P_{R x}=\oint_{\Omega_{R x}} P_{D}^{\prime}(\phi, \theta) \sin (\theta) d \theta d \phi
$$

\section{Power Loss due to Human Body Shadowing}

The APD model shows the power of the incoming waves considering certain power distribution in a spatial area centered at the receiver. Therefore, with the movement of people around the radio link, at certain position, the human body can shadow certain angular area cutting the incoming waves. This essentially affects the signal reception and reduces the received power. We consider people moving horizontally on the ground that is parallel to $\mathrm{X}-\mathrm{Y}$ plane. The body of a person is modeled as a cylinder with height $H_{P}$ and radius $W_{P}$. We separate the 3dimensional coordinate system into the $\mathrm{X}-\mathrm{Y}$ and the $\mathrm{X}-\mathrm{Z}$ planes as shown in Fig. 2. Accordingly, the position of person is represented as $\left(X_{R x P}, Y_{R x P},-H_{R x}\right)$, with $H_{R x}$ being the receiver's height. Consider the LOS direction along the $\mathrm{X}$-axis and the transmitter's position as $\left(D_{t r}, 0,0\right)$ with $D_{t r}>X_{R x P}>0$ as illustrated in Fig. 2, the shadowed azimuth area and elevation area are bounded, respectively by:

$$
\begin{aligned}
\phi_{s}^{\min }= & \arctan \left(\frac{Y_{R x P}}{X_{R x P}}\right)-\arctan \left(\frac{W_{P}}{\sqrt{X_{R x P}^{2}+Y_{R x P}^{2}}}\right), \\
\phi_{s}^{\max }= & \arctan \left(\frac{Y_{R x P}}{X_{R x P}}\right)+\arctan \left(\frac{W_{P}}{\sqrt{X_{R x P}^{2}+Y_{R x P}^{2}}}\right), \\
& \left\{\begin{array}{l}
\theta_{s}^{\min }=\frac{\pi}{2}-\arctan \left(\frac{H_{P}-H_{R x}}{X_{R x P}}\right), \\
\theta_{s}^{\max }=\frac{\pi}{2}+\arctan \left(\frac{H_{R x}}{X_{R x P}}\right) .
\end{array}\right.
\end{aligned}
$$

At time $t$, the power loss due to this shadowing is related to the angular area of the shadowing at that moment, and can be expressed as,

$$
P_{L_{S}}(t)=\int_{\phi_{S}^{\min }(t)}^{\phi_{S}^{\max }(t)} \int_{\theta_{S}^{\min }(t)}^{\theta_{\max }(t)} P_{D}^{\prime}(\phi, \theta) \sin (\theta) d \theta d \phi .
$$

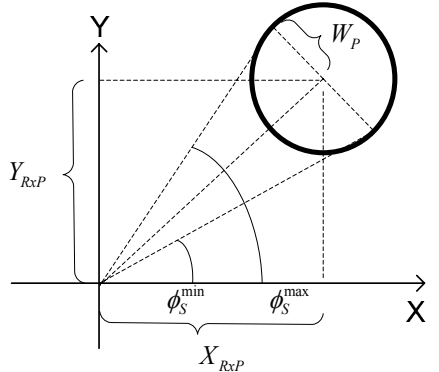

(a)

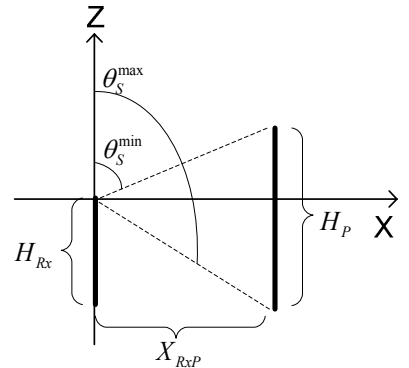

(b)
Fig. 2. The shadowing angular area of the human body: (a) on azimuth $(\mathrm{X}-\mathrm{Y})$ plane, and (b) on elevation (X-Z) plane.

Consider the simple case when the transmitter and the receiver have the same height and are located at $(10,0,0)$ and $(0,0,0)$ respectively. Based on (9)-(11) we are able to calculate the power loss when a person stands at various places between the transmitter and the receiver cutting the LOS as well as reflected waves. In Fig. 3 we have plotted the power loss in the cases that the transmitter and the receiver both have omnidirectional and both have directional antennas. The parameters are set as given in Table 1. The values of $K, \tau_{\max }$ and $\gamma$ refer to the measurements given in [21]. Shown in Fig. 3, when the person is closer to the LOS and closer to the receiver as well, the power loss increases for both omni-directional and directional antenna configurations. A large amount of power loss is observed when the person is close to the receiver. For example, when the distance is less than $1 \mathrm{~m}$, power loss in the omni-directional case is more than $10 \mathrm{~dB}$ and in the directional case more than $20 \mathrm{~dB}$. When the person is not intersecting the LOS, i.e., $Y_{R x P}>0.3$ or $Y_{R x P}<-0.3$, the power loss is marginal, 


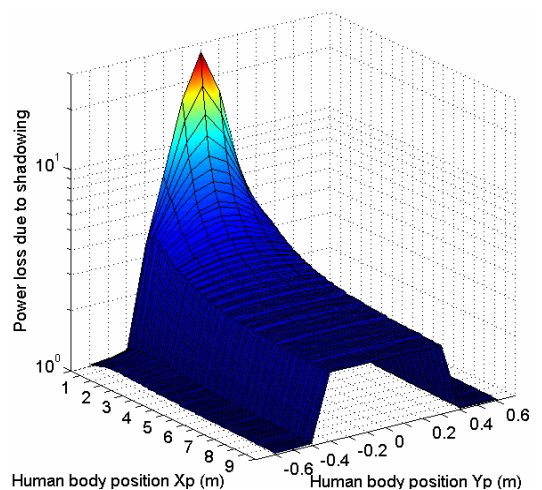

(a)

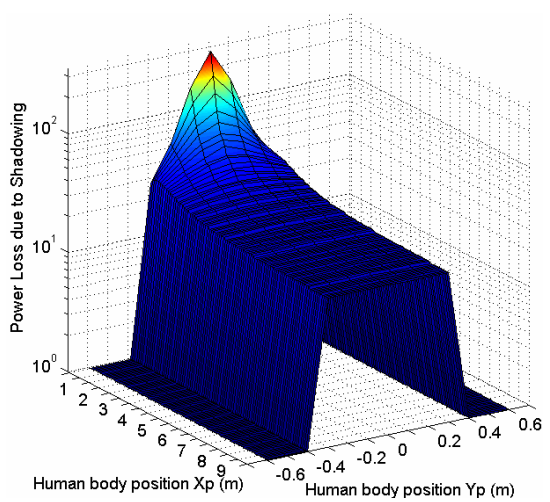

(b)

Fig. 3. Shadowing loss of a person in different positions: (a) omnidirectional antennas, (b) directional antennas taking $q=2$.

close to $0 \mathrm{~dB}$ for both cases. Comparatively, the human shadowing has much severe impact while using the directional antenna. Shadowing of LOS wave leads to at least $10 \mathrm{~dB}$ power loss depending on the distance from the receiver. While for omni-directional case, only $2-3 \mathrm{~dB}$ loss occurs when person is away from the receiver.

\section{Link Stability Calculation}

\section{Link Budget Analysis}

Link budget accounts all of the power gains and losses from the transmitter through the medium to the receiver. The calculation is used for checking if the required minimum signal-to-noise ratio (SNR) is met for the receiver to achieve demodulation and decoding for different constellations. SNR can be calculated from the received signal power as,

$$
\Gamma(t)=P_{R x}-N_{0}-L_{I},
$$

where $N_{0}=-174+10 \lg B+F$ is the thermal noise level in $\mathrm{dB}$ at the standard temperature $17^{\circ} \mathrm{C}$ with the bandwidth $B$ in $\mathrm{Hz}$ and the noise figure $F$ in $\mathrm{dB} . L_{I}$ is the implementation loss of the transceiver. From link budget calculation, the received signal power is,

$$
P_{R x}=P_{t}+G_{T x}+G_{R x}-P L(d)-P_{L_{s}}(t),
$$

where $P_{t}$ is the transmission power, $G_{T x}, G_{R x}$ are the antenna gain of the transmitter and the receiver respectively. $P L(d)$ is the path loss for indoor environment, and it follows the lognormal model,

\begin{tabular}{|c|c|c|c|}
\hline Variable & Unit & Value & Description \\
\hline \multirow{2}{*}{$K$} & 1.1 & \multirow{2}{*}{ - } & Rician factor (omni) \\
\hline & 1.7 & & Rician factor (directional) \\
\hline \multirow{2}{*}{$\gamma$} & 0.2 & \multirow{2}{*}{$\mathrm{dB} / \mathrm{ns}$} & Decay factor (omni) \\
\hline & 0.48 & & Decay factor (directional) \\
\hline \multirow{2}{*}{$\tau_{\max }$} & 67.8 & \multirow{2}{*}{ ns } & Max. multipath delay (omni) \\
\hline & 15.7 & & Max. multipath delay (directional) \\
\hline$q$ & 2 & - & Directional antenna power pattern index \\
\hline$P_{t}$ & 10 & $\mathrm{dBm}$ & Transmission power \\
\hline$G_{T x}, G_{R x}$ & 10 & $\mathrm{dBi}$ & Directional antenna gain \\
\hline \multirow{2}{*}{$P L\left(d_{0}\right)$} & -70 & \multirow{2}{*}{$\mathrm{dB}$} & Path loss reference at $1 \mathrm{~m}$ (omni) \\
\hline & -67 & & Path loss reference at $1 \mathrm{~m}$ (directional) \\
\hline$n$ & 2.1 & - & Path loss exponent \\
\hline$X_{\Omega}$ & 0.8 & $\mathrm{~dB}$ & Gaussian noise \\
\hline$L_{I}$ & 2.5 & $\mathrm{~dB}$ & Implementation loss \\
\hline$B$ & 1.28 & $\mathrm{GHz}$ & Bandwidth \\
\hline$F$ & 7 & $\mathrm{~dB}$ & Noise figure \\
\hline$H_{P}$ & 1.8 & $\mathrm{~m}$ & Average people height \\
\hline$W_{P}$ & 0.3 & $\mathrm{~m}$ & Average people width \\
\hline$H_{T x}, H_{T x}$ & 0.9 & $\mathrm{~m}$ & Antenna height \\
\hline$D_{t r}$ & $2,5,10$ & $\mathrm{~m}$ & Distance between Antennas \\
\hline $\mathcal{A}$ & 100 & $\mathrm{~m}^{2}$ & Room size \\
\hline$v_{\min }$ & 0.5 & $\mathrm{~m} / \mathrm{s}$ & Min. walking speed \\
\hline$v_{\max }$ & 2 or 5 & $\mathrm{~m} / \mathrm{s}$ & Max. walking speed \\
\hline \multirow{2}{*}{$\Gamma^{t h}$} & 11.7 & \multirow{2}{*}{$\mathrm{dB}$} & "Min. required SNR (QPSK) \\
\hline & 15 & & Min. required SNR (8PSK) \\
\hline \multirow{6}{*}{$P_{L_{s}}^{t h}$} & 2.9 & \multirow{6}{*}{$\mathrm{dB}$} & Max. shadowing loss (10 m, QPSK) \\
\hline & - & & Max. shadowing loss $(10 \mathrm{~m}, 8 \mathrm{PSK})$ \\
\hline & 9.2 & & Max. shadowing loss (5 m, QPSK) \\
\hline & 5.9 & & Max. shadowing loss (5 m, 8PSK) \\
\hline & 17.5 & & Max. shadowing loss (2 m, QPSK) \\
\hline & 14.2 & & Max. shadowing loss ( $2 \mathrm{~m}, 8 \mathrm{PSK})$ \\
\hline
\end{tabular}

Table 1. Parameters Setting

$$
P L(d)=P L\left(d_{0}\right)+10 n \log \left(\frac{d}{d_{0}}\right)+X_{\Omega},
$$

where $P L\left(d_{0}\right)$ is the reference path loss measured when the transmitter and the receiver are of $d_{0}=1 \mathrm{~m}$ apart; $n$ is the loss exponent depending on the environment; $X_{\Omega}$ is the zero mean Gaussian noise with the standard deviation $\Omega$. For the minimum required SNR, the shadowing loss due to the presence of people close to the transmission link should be below a maximum value, i.e.,

$$
P_{L_{s}}^{t h}=P_{t}+G_{T x}+G_{R x}-P L(d)-\left(\Gamma^{t h}+N_{0}+L_{I}\right) .
$$

The parameters for calculating the maximum shadowing loss are listed in Table 1, where the values of $P L\left(d_{0}\right), n$ and $X_{\Omega}$ are taken from the measurement results given in [21] and [24] for omni-directional transceivers and directional transceivers, respectively. The value of $\Gamma^{\text {th }}$ is taken from [25] for an OFDM system with ideal front-end, achieving BER of $10^{-5}$ in Rayleigh channels. We calculate and list the values of $P_{L_{s}}^{\text {th }}$ in Table 1. From the link budget calculation we find that the 
omni-directional case cannot fulfill the SNR requirement for QPSK and 8PSK to achieve data rate above Giga bits per second. Therefore only the directional case is listed. Even for the directional antenna configuration when the distance between the transmitter and the receiver is $10 \mathrm{~m}$, it is hard to achieve the required SNR for using 8PSK.

\section{Link Stability Model}

To reflect on the ability of the link to sustain certain link quality to support QoS requirement, we have developed a link stability model by investigating the statistics of link failure duration, which is under a given modulation scheme and determined by the received SNR. As the SNR is affected by the position of people around the link, the statistics of link failure is influenced by the movement pattern of people.

As we can see in Table 1, for the given modulation and distance to have enough SNR preventing link failure, people should stay outside the area that results in the corresponding maximum shadowing loss. Based on the power loss calculation for the directional antenna configuration as shown in Fig. 3(b), we have plotted in Fig. 4 the contours of the areas, which when people stay outside there is enough SNR to support the receiver processing given the considered modulations, i.e., QBSK or 8PSK. In this sense, for certain modulation the link failure duration is the period of time the people stay inside the contour enclosed area that corresponds to the maximum shadowing loss tolerable for the modulation scheme used.

Theoretically, given the contour shape and the mobility model of people, the distribution of the duration that a person stays in the contour enclosed area can be found. However, with irregular contour shapes and complex mobility models a closed form equation is always hard to derive. Thus we first discuss the link failure statistics using the random way point (RWP) mobility model, for which the parameters are adjusted from the field measurements to create the realistic scenarios. We choose to use RWP since RWP is designed to capture the significant mobility feature of the mobile nodes and is commonly used to study various aspects of wireless mobile networks. To direct the analytical result we further consider the case with certain simplifications where the distribution of the failure duration is mathematically derivable.

1) Simulation Based on RWP: Here we further investigate, using RWP with the adjusted parameters, the influence of the movement of people on link stability in realistic scenarios. In [26] the spatial distribution of the mobile nodes with RWP is given as,

$$
f_{X Y}(x, y)=p_{P} f_{X Y, P}(x, y)+\left(1-p_{P}\right) f_{X Y, M}(x, y),
$$

where $p_{P}$ denotes the probability that a given node pauses at a randomly chosen time, and is given by the percentage of the total duration that a node pauses during a long-running process. Referring to [26], in the square area of $(a \times a) \mathrm{m}^{2}$ and with the average speed $E[v]$ while moving, $p_{P}$ can be expressed as $p_{P}=\frac{E\left[T_{P}\right]}{E\left[T_{P}\right]+E\left[T_{M}\right]}=\frac{\frac{E[v]}{a} E\left[T_{P}\right]}{\frac{E[v]}{a}+0.5214}$, with $E\left[T_{P}\right]$ being the mean pause duration and $E\left[T_{M}\right]$ the mean moving duration. $f_{X Y, P}(x, y)$ represents the spatial distribution of all nodes that are currently pausing at a destination point, which by definition of RWP has a uniform spatial distribution as $f_{X Y, P}(x, y)=\left\{\begin{array}{ll}1 / \mathcal{A}, & (\mathrm{x}, \mathrm{y}) \in \mathcal{A}, \\ 0, & \text { else, }\end{array}\right.$ in the area of size $\mathcal{A}$. $f_{X Y, M}(x, y)$ represents the spatial distribution of all moving nodes. In a square area of size $\mathcal{A}=a \times a$, and $-a / 2 \leq x \leq a / 2$, $-a / 2 \leq y \leq a / 2$, it can be approximated as $f_{X Y, M}(x, y) \approx 36 / a^{6}\left(x^{2}-a^{2} / 4\right)\left(y^{2}-a^{2} / 4\right)$.

For certain modulation, the probability of the link failure due to shadowing loss led by the presence of people around the communication link is

$$
\pi_{c}=\iint_{(x, y) \in \mathcal{A}} f_{X Y}(x, y) d x d y,
$$

where $\mathcal{A}_{c}$ is the size of the area where the presence of people leads to SNR degrading below the minimum required value for using the given modulation (see Table 1).

As RWP results in non-uniform spatial distribution, the location of the transmitter and the receiver affects the chance of a given link being interrupted due to the movement of people. Let us consider the communication link to be in the middle of the room. To find a proper value of $p_{P}$, it is important to determine the values of $E[v]$ and $E\left[T_{P}\right]$. For this propose we have carried out field measurements in a student computer room by deploying a pair of Infra red (IR) transceivers of $5 \mathrm{~m}$ apart. In the natural scenarios we observed that the ratio of the period that people block the IR LOS link is about 3\%. Further we have built a Matlab RWP simulator. We create a scenario in which a room of $10 \times 10 \mathrm{~m}^{2}$ is considered and, the transmitter and receiver are placed $5 \mathrm{~m}$ apart in the way that the LOS link is in the middle of the room and parallel to one edge of the room. Considering the average walking speed of 2-3 mph [27], we assumed the speed of movement of people, in our simulation, to be uniformly distributed in the range $\left[v_{\min }=0.5, v_{\max }=2\right](\mathrm{ms})$. We also assumed the pause duration to be uniformly distributed in the range $\left[0, T_{P_{\max }}\right]$. We tested $T_{P \max }$ with different values in the simulation, and based on that we take $T_{P \max }=540$ s (i.e., $E\left[T_{P}\right]=270 \mathrm{~s}$ ) resulting in $p_{P} \approx 0.98$, which gives the LOS blockage ratio close to the measurement result of 3\%. Using the parameter set in Table 1, when the distance between the transmitter and the receiver takes values of $10 \mathrm{~m}, 5 \mathrm{~m}$ and $2 \mathrm{~m}$, the probability of experiencing a link failure using QPSK is $6.89 \%, 2.70 \%$ and $0.18 \%$ respectively. For $8 \mathrm{PSK}$, when the distance between the transceiver pair is as far as $10 \mathrm{~m}$, the path loss is too severe to achieve the received SNR which is enough for the modulation. For the distances $5 \mathrm{~m}$ and $2 \mathrm{~m}$, the probability of experiencing a link failure using $8 \mathrm{PSK}$ is $2.91 \%$ and $0.62 \%$ respectively.

Fig. 5 plots the simulation results of CDF of link failure duration and link failure interval using the adjusted RWP. In 


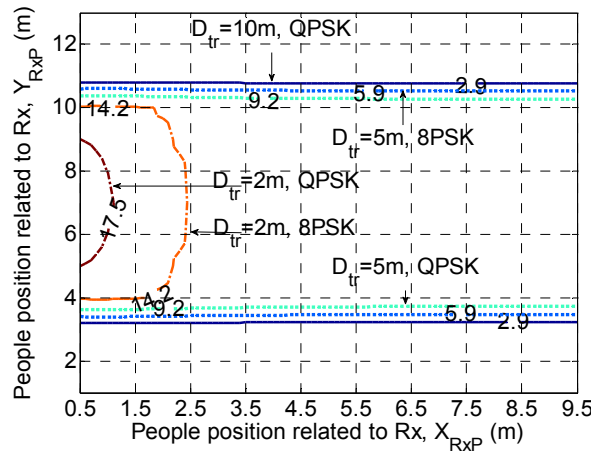

Fig. 4. Contour plot of power loss due to human shadowing marking different position.

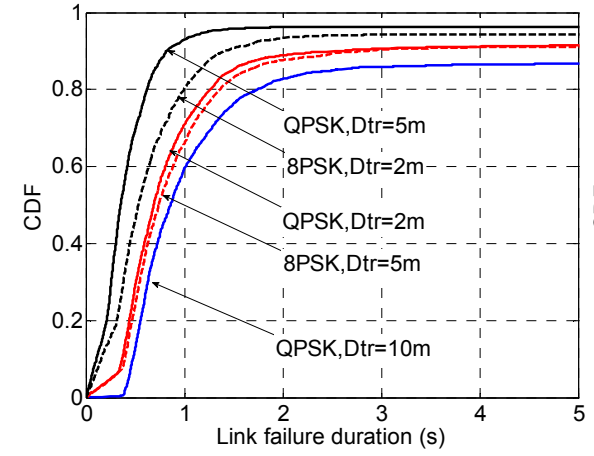

Fig. 5(a). RWP Simulation results: CDF of link failure duration.

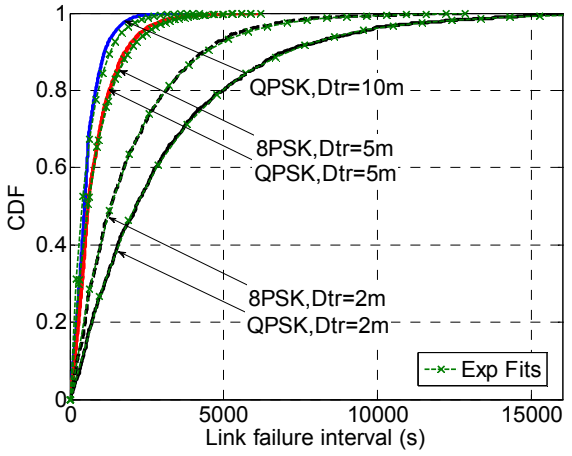

Fig. 5(b). RWP Simulation results: CDF of link failure interval.
Fig. 5(a) the link failure probability increases with $D_{t r}$. This is due to the fact that bigger the distance, the smaller is the maximum tolerable shadowing loss. Further, it results in a bigger area where people may enter and break the link. At a given distance, 8PSK requires higher SNR than QPSK, and thus is more vulnerable to the movement of people around. Besides we observed a low chance of occurrence of long link failure, e.g., over $5 \mathrm{~s}$, which is due to people pausing in the positions that cause link failure. Fig. 5(b) shows the CDF of the link failure interval. We have tried fitting various distributions for each case. We found that the CDF of link failure interval obtained through simulation agrees well with the corresponding exponential distribution fit, especially, when the distance between the transmitter and the receiver is smaller, as shown in Fig. 5(b).

2) Analysis Based on Simplification: Due to the complexity of the RWP mobility model, analytical result is hard to achieve. Thus we design the simplified case where the distribution of the failure duration is mathematically derivable. This is favorable for many studies that can use the link stability as the input for further analysis. Specifically, we consider a simplified case with the following assumptions:

(1) Referring to Fig. 4, we approximate the contour lines into rectangular shape, of which the size is $\mathcal{A}_{c}=w \times l$. Here $w$ is the length of the edge perpendicular to the LOS direction, and $l$ is the length of the edge parallel to the LOS direction.

(2) From the simulations with RWP mobility model, the link failure intervals can be modeled with exponential distribution. We assume the occurrence of disturbances due to people moving in the contour area as a Poisson process. The probability of a person causing a significant shadowing is $\rho$, which is the ratio of the size of the contour enclosed area to the size of the room where the transmitter and the receiver are placed, i.e., $\rho=\mathcal{A}_{c} / \mathcal{A}$. For $N$ people with independent mobility, the probability of crossing LOS is $N \rho$.

(3) The entering direction, $\alpha$ is perpendicular to the edge from which the person enters the contour area and causes a significant shadowing, i.e., $\alpha=\pi / 2$.

(4) After entering the contour enclosed area, we assume that the person keeps moving perpendicular to the LOS direction before leaving the area. Thus the moving distance of the person inside the contour area is $w$.

(5) After entering the contour enclosed area, we assume that the person has a constant speed while passing through the area. The speed is uniformly distributed within $\left[v_{\min }, v_{\max }\right]$.

Based on the above assumptions, the link failure duration is $t_{d}=\frac{w}{v}$, where the probability density function (PDF) of the speed is $f_{V}(v)=1 /\left(v_{\max }-v_{\min }\right)$. PDF and CDF of the link failure duration are derived, respectively as:

$$
\begin{gathered}
f_{T_{d}}\left(t_{d}\right)= \begin{cases}\frac{w}{t_{d}^{2}\left(v_{\max }-v_{\min }\right)}, & \frac{w}{v_{\max }} \leq t_{d} \leq \frac{w}{v_{\min }}, \\
0, & \text { Otherwise. }\end{cases} \\
F_{T_{d}}\left(t_{d}\right)= \begin{cases}0, & 0<t_{d}<\frac{w}{v_{\max }}, \\
\frac{v_{\max }}{v_{\max }-v_{\min }}-\frac{w}{t_{d}\left(v_{\max }-v_{\min }\right)}, & \frac{w}{v_{\max }} \leq t_{d} \leq \frac{w}{v_{\min }}, \\
1, & \frac{w}{v_{\min }}<t_{d} .\end{cases}
\end{gathered}
$$

Fig. 6 shows the comparison of link stability while using different modulations and for the transmitter and the receiver being placed with various distances in between. Using the values of the parameters given in Table 1, we have first calculated $\rho$ according to the second assumption. For QPSK, $\rho$ is $6.81 \%, 2.64 \%$ and $0.18 \%$ when the distance of the link is $10 \mathrm{~m}, 5 \mathrm{~m}$ and $2 \mathrm{~m}$ respectively; and for $8 \mathrm{PSK}$, it is $2.84 \%$ and $0.61 \%$ for distance of $5 \mathrm{~m}$ and $2 \mathrm{~m}$. This result agrees well with the values from RWP simulation. Thus we can claim that using the ratio of contour area to the room area is a good approximation for the probability of having a link failure. Further Fig. 6 shows that the link failure probability is consistent with the observation from the RWP simulation. That is, the link stability is better if the distance between the transmitter and the receiver is shorter. The link is more stable for QPSK than for 8PSK. Moreover, it shows for any modulation at a given distance, with higher speed, i.e., when 


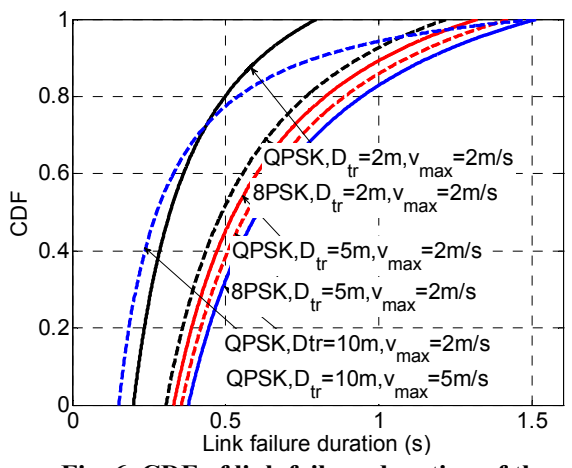

Fig. 6. CDF of link failure duration of the simplified mobility model, $F_{T_{d}}\left(t_{d}\right)$.

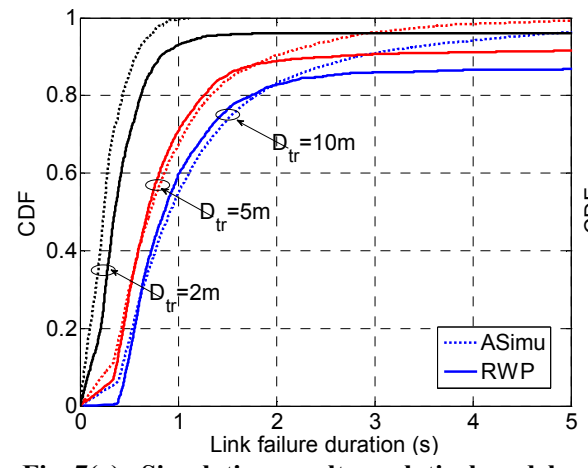

Fig. 7(a). Simulation result: analytical model vs. RWP with pause in link failures.

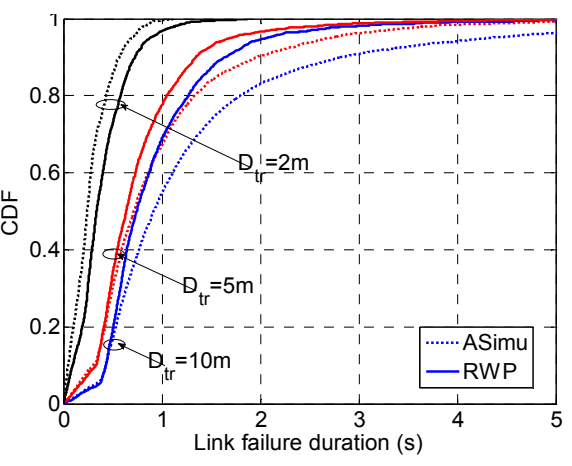

Fig. 7(b). Simulation result: analytical model vs. RWP without pause in link failures. people pass through the contour faster, the link stability is better, which can be easily expected.

However, by comparing Fig. 6 with Fig. 5(a) we observe certain inconsistency between the corresponding curves due to the simplification of the movement pattern for the analysis. Especially since we assume that a person crosses the LOS perpendicularly, the minimum link failure duration for each case is, rather than 0 , but a value determined by the approximated edge length $w$ and the maximal walking speed $v_{\max }$. Thus we relaxed the assumptions in the model as that the person enters and leaves the contour enclosed area (approximated as a rectangle) and cutting the LOS path from two arbitrary points on two different edges on the contour. The entering direction and speed are uniformly distributed within $(0, \pi)$ and $\left[v_{\min }, v_{\max }\right]$ respectively. After entering, we assume that the person keeps moving in the same direction at the same speed until leaving the area. This is reasonable since the contour enclosed areas are in general of smaller size and the duration the person moving across LOS is short. Thus the possibility of changing the movement pattern in a small area and in a short duration is also small. We use $x_{l}$ and $x_{w}$ to represent the entry point when the person enters the contour area from the edges parallel and perpendicular to the LOS direction, and $x_{l}$ and $x_{w}$ are uniformly distributed within $[0, l]$ and $[0, w]$ respectively. We can formulate the link failure duration into (21) and (22), which are corresponding to the person entering from $x_{l}$ and $x_{w}$ respectively.

We have used Matlab to generate the random variables $\alpha, v, x_{l}$ and $x_{w}$, and calculated the corresponding link failure durations. The distribution of the link duration has thus been derived using the observed link failure samples from the simulations. We argue here that the closed form equation for link failure duration distribution is analytically derivable, however it is cumbersome as the link failure duration is a function of three random variables, $\alpha, v$ and $x_{l}$ (or $x_{w}$ ). We compare in Fig. 7 the results of the analytical model (by simulation) and RWP model for different distances when using QPSK. Fig.7 (a) shows that the curves under the analytical model have similar shapes with the ones from
RWP, especially

$$
\begin{aligned}
& \int \frac{x_{l}}{v \cos (\alpha)}, 0<\alpha \leq \arctan \left(\frac{w}{x_{l}}\right), \\
& t_{d}^{(l)}=\left\{\frac{w}{v \sin (\alpha)}, \arctan \left(\frac{w}{x_{l}}\right)<\alpha \leq \arctan \left(\frac{w}{l-x_{l}}\right),\right. \\
& \frac{l-x_{l}}{-v \cos (\alpha)}, \arctan \left(\frac{w}{l-x_{l}}\right)<\alpha<\pi \text {. } \\
& \int \frac{x_{w}}{v \cos (\alpha)}, 0<\alpha \leq \arctan \left(\frac{l}{x_{w}}\right) \\
& t_{d}^{(w)}=\left\{\frac{l}{v \sin (\alpha)}, \arctan \left(\frac{l}{x_{w}}\right)<\alpha \leq \arctan \left(\frac{l}{w-x_{w}}\right),\right. \\
& \frac{w-x_{w}}{-v \cos (\alpha)}, \arctan \left(\frac{l}{w-x_{w}}\right)<\alpha<\pi,
\end{aligned}
$$

for the transceiver distance of $10 \mathrm{~m}$ and $5 \mathrm{~m}$. The inconsistency is more prominent at the two ends of the curves. At the high end, the difference is mainly due to the RWP including pauses when people stand in positions that lead to link failures. The lower end difference can be explained as the side effect of the pauses, as the existence of the long link failure duration reduces the percentage of the short ones. As shown in Fig. 7(b), we plot the CDF of link duration of RWP model but subtracting the pause duration when the person stands in the positions causing a link failure. From Fig. 7(b), we see the lower ends of the curves from both analytical model and RWP model agree well. Thus this analytical model is able to describe distribution of link failures with the moderate duration ( 0.5 to $2 \mathrm{~s}$ in our presented case) under RWP, or with the short duration (below $0.5 \mathrm{~s}$ ) under RWP excluding the case that the person pauses in the positions causing link failures.

\section{Conclusion}

Our ray-based model is a feasible approach to model the human body shadowing effects on the received signal strength and in turn it allows us to estimate the link stability, which is very dependent on the availability of LOS. Thus link stability is affected by the movement of people in the room. Due to the complicated movement pattern of people, we have studied the $60 \mathrm{GHz}$ link stability in the context of RWP mobility model after adjusting the critical parameters using the simulation. 
This can be considered as a practical approach for modeling the influence of the presence of people on indoor $60 \mathrm{GHz}$ radio links. We have also investigated an approach which is able to describe the distribution of link failure duration with an analytical expression - in our case with certain simplifications. The closed form expression has been presented for the simplified analytical model.

The proposed link stability model is flexible enough and extensible to reflect the capability for the link to sustain different QoS requirements under different channel conditions, antenna configurations and movement patterns of people. The link stability model quantified by the probability of link failure below a certain threshold is a promising input in designing and analyzing many other protocols and algorithms. The complete end-to-end analytical use of link stability is yet to come. To have a complete picture of the effect of movements of persons in an indoor environment with $60 \mathrm{GHz}$ links, we need to evaluate, for example, routing algorithms for applications such as multimedia streaming. Though we do not claim to spell the last words with the study presented here, we believe that the results obtained here are far satisfactory and assuring.

\section{REFERENCES}

[1] EBU - tech 3299: High definition (hd) image formats for television production. [Online]. Available: http://www.ebu.ch/CMSimages/en/tec doc $\mathrm{t} 3299$ tcm6-23327.pdf

[2] ISO/IEC 14496-10:2009 information technology - coding of audiovisual objects - part 10: Advanced video coding. [Online]. Available: http://www.iso.org/iso/iso catalogue/catalogue ics/ catalogue detail ics.htm? csnumber $=52974$

[3] The WirelessHD specification. [Online]. Available: http://www. wirelesshd.org/company/about.html

[4] IEEE 802.15 WPAN Task Group 3c - millimeter wave alternative phy. [Online]. Available: http://www.ieee802.org/15/pub/TG3c.html

[5] Ecma tc48 - high rate wireless communications. [Online]. Available: http://www.ecma-international.org/memento/TC48-M.htm

[6] N. Moraitis and P. Constantinou, "Indoor channel measurements and characterization at $60 \mathrm{ghz}$ for wireless local area network applications", IEEE Trans. Antennas Propagat., vol. 52, no. 12, pp. 3180-3189, 2004.

[7] R. Dube, C. Rais, K.-Y. Wang, and S. Tripathi, "Signal stability based adaptive routing (ssa) for ad hoc mobile networks," IEEE Personal Commun. Mag., vol. 4, no. 1, pp. 36-45, 1997.

[8] G. Lim, K. Shin, J.-S. Kim, and H. Yoon, "Signal strength-based link stability estimation in ad hoc wireless networks," Electronics Letters, vol. 39 , no. 5, pp. 485-486, 2003.

[9] C.-K. Toh, "Associatively-based routing for ad hoc mobile networks," IEEE Personal Commun. Mag., vol. 4, no. 2, pp. 1-36, 1997.

[10] M. Gerharz, C. de Waal, M. Frank, and P. Martini, "Link stability in mobile wireless ad hoc networks," in Proc. IEEE LCN, US-FL, 2002.

[11] I. Rubin and Y. Liu, "Link stability models for QoS ad hoc routing algorithms," in Proc. IEEE VTC'03-Fall, Orlando, US-FL, 2003.

[12] M.-G. Lee and S. Lee, "A link stability model and stable routing for mobile ad-hoc networks," LNCS, vol. 4096/2006, pp. 904-913, 2006.

[13] K. Sridhar and L. Jacob, "Performance evaluation and enhancement of a link stability based routing protocol for manets," IJHPCN, vol. 4, no. 1/2, pp. 66-77, 2006.

[14] L. Meng and W. Wu, "Dynamic source routing protocol based on link stability arithmetic," in Proc. ISISE'08, Shanghai, China, 2008.

[15] S. Collonge, G. Zaharia, and G. El Zein, "Influence of human activity on wide-band characteristics of the $60 \mathrm{ghz}$ indoor radio channel," IEEE Trans. Wireless Commun., vol. 3, no. 6, pp. 2396-2406, Nov. 2004.

[16] K. Sato and T. Manabe, "Estimation of propagation-path visibility for indoor wireless lan systems under shadowing condition by human bodies," in Proc. IEEE VTC'98, Ottawa, Canada, 1998.
[17] S. Collonge, G. Zaharia, and G. El Zein, "A body-shadowing model for indoor radio communication environments," IEEE Trans. Antennas Propagat., vol. 46, no. 6, pp. 920-927, June 1998.

[18] R. Zhang and L. Cai, "A markov model for indoor ultra-wideband channel with people shadowing," Mobile Network Applications, vol. 12, pp. 438-449, 2007.

[19] W. Fu and J. Ma, "Improving the accuracy of two-ray model at $60 \mathrm{GHz}$ for line-of-sight wireless desktop channel," Microwave and Optical Technology Letters, vol. 51, no. 4, pp. 907-910, Feb. 2009.

[20] N. Azzaoui and L. Clavier, "An impulse response model for the $60 \mathrm{GHz}$ channel based on spectral techniques of a-stable processes," in Proc. IEEE ICC '07, Glasgow, Scotland, 2007.

[21] H. Yang, P. Smulders, and M. Herben, "Channel characteristics and transmission performance for various channel configurations at 60 GHz," EURASIP Journal of Wireless Communications and Networking, vol. 2007, 2007

[22] H. Yang, M. Herben, I. Akkermans, and P. Smulders, "Impact analysis of directional antennas and multiantenna beamformers on radio transmission," IEEE Trans. Veh. Technol., vol. 57, no. 3, pp. 16951707, May 2008.

[23] A. F. Molisch, "Time variance for UWB wireless channels," 2002, IEEE P802.15-02/461-SG3a Standard Contribution.

[24] C. Anderson and T. Rappaport, "In-building wideband partition loss measurements at 2.5 and $60 \mathrm{ghz}$," IEEE Trans. Wireless Commun., vol. 3, no. 3, pp. 922-928, May 2004.

[25] U. H. Rizvi, G. J. Janssen, and J. H. Weber, "Impact of RF circuit imperfections on multi-carrier and single-carrier based transmissions at $60 \mathrm{GHz}$," in Proc. IEEE RWS'08, Orlando, US-FL, 2008.

[26] C. Bettstetter, H. Hartenstein, and X. Prez-Costa, "Stochastic properties of the random waypoint mobility model," Wireless Networks, vol. 10, no. 5, pp. 555-567, Sept. 2004.

[27] R. Gross and J. Shi, "The CMU motion of body (mobo) database," 2001, technical report, Robotics Institute, Carnegie Mellon University.

\section{BIOGRAPHIES}

Jing Wang received her BSc degree in Electrical Engineering from Beijing University of Aeronautics and Astronautics, China, in 2003. In 2005 she received MSc degree on Telecommunications from Delft University of Technology, the Netherlands. She is now a PhD student in Wireless and Mobile Networks (WMC) group in Delft University of Technology, and working on Dutch IOP GenCom "Future Home Networks" project. Her present research interests are wireless mesh networks, high data rate wireless personal networks, wireless sensor networks, cognitive networking and selforganization systems.

R. Venkatesha Prasad received bachelor's degree in Electronics and Communication Engineering from the University of Mysore, India, in 1991. In 1994 he received a M.Tech degree in Industrial Electronics and a Ph.D. degree in 2003 from University of Mysore, India and Indian Institute of Science, Bangalore, India. From 2003 to 2005 he was heading a team of engineers at the Esqube Communication Solutions Pvt. Ltd., Bangalore, India, for the development of various networking solutions. From 2005 until date he is with the Wireless and Mobile Communications Group, Delft University of Technology, the Netherlands. He is IEEE 1900 Standardization committee member and member of IEEE TCCC, TCCN and AHSNTC. He has been the tutorial co-chair for CCNC, Organizing committee member for PerNets-2006, 2007. He has also been the chair of Cognet 2007, 2008, 2009.

Ignas G.M.M. Niemegeers got a degree in electrical engineering from the University of Gent, Belgium, in 1970. In 1972 he received an M.Sc.E. degree in computer engineering and in 1978 a Ph.D. degree from Purdue University in West Lafayette, Indiana, U.S.A. From 1978 to 1981 he was a designer of packet switching networks at Bell Telephone Mfg. Cy, Antwerp, Belgium. From 1981 to 2002 he was a professor at the Computer Science and the Electrical Engineering Faculties of the University of Twente, Enschede, the Netherlands. Since May 2002 he holds the chair Wireless and Mobile Networks at Delft University of Technology, where he is heading the Centre for Wireless and Personal Communication (CWPC). He is an active member of the Wireless World Research Forum (WWRF) and IFIP TC-6 Working Group on Personal Wireless Communication. 